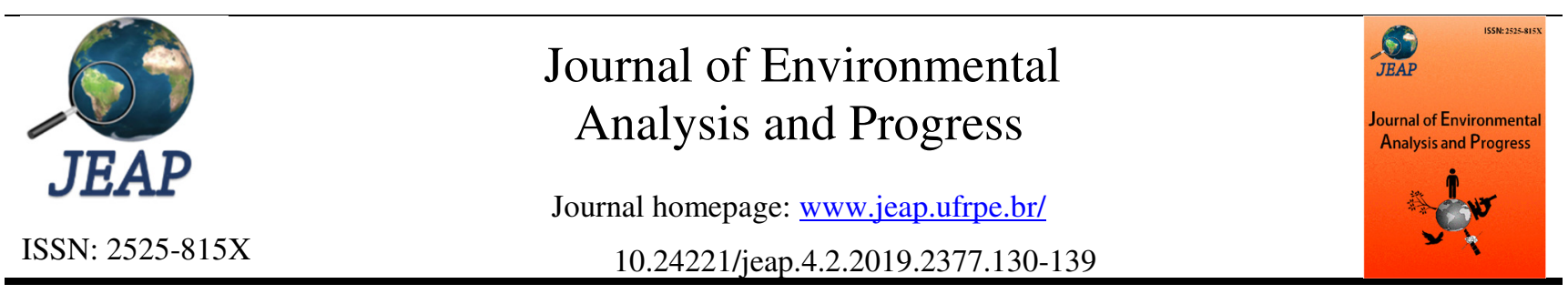

\title{
Análise de tendência para dados pluviométricos no município de Toritama-PE
}

\section{Trend analysis for pluviometric data in the municipality of Toritama-PE (Brazil)}

Lucas Ravellys Pyrrho de Alcântara ${ }^{\mathrm{a}}$, Ialy Rayane de Aguiar Costa ${ }^{\mathrm{b}}$, Vitor Hugo de Oliveira Barros $^{\mathrm{c}}$, Severino Martins dos Santos Neto ${ }^{\mathrm{a}}$, Artur Paiva Coutinho ${ }^{\mathrm{d}}$, Antônio Celso Dantas Antonino $^{\mathrm{a}}$

a.Universidade Federal de Pernambuco-UFPE, Departamento de Energia Nuclear-DEN, Programa de Pós-Graduação em Tecnologias Energéticas e Nucleares-PROTEN. Av. Luiz Freire, n. 1000. Cidade Universitária, Recife-PE, Brasil. CEP: 50740-545.E-mail: ravellyspyrrho@gmail.com, martinsdsn@gmail.com, acdantonino@gmail.com.

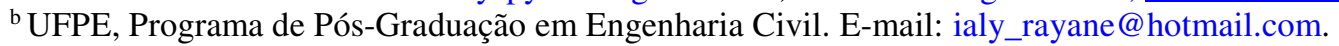

c.UFPE, Programa de Pós-Graduação em Engenharia Civil e Ambiental. E-mail: vitor_barros1@outlook.com.

${ }^{\mathrm{d}}$ UFPE, Centro Acadêmico do Agreste. E-mail: arthur.coutinho@yahoo.com.br.

A R T I C L E I N F O

Recebido 04 Fev 2019

Aceito 25 Fev 2019

Publicado 28 Fev 2019

\begin{abstract}
A B S T R A C T
The atmospheric changes caused by development and industrialization process directly affect the hydrological cycle. The knowledge of tendency in time series of hydrological data has a scientific and practical importance for the management of water resources. The objective of this study is to investigate tendency and fluctuations over time in climatological variables (annual maximum daily precipitation, total annual rainfall, days without rainfall, and precipitation of the seasons) in Toritama-PE. The Mann-Kendall test and the Sen's Slope ( $\beta$ ) estimator were used to determine the existence of tendency and its magnitude. Mann-Kendall sequential test was used to identify fluctuations in tendency over time and to detect possible points of change in the rainfall series. The non-parametric Mann-Kendall test showed no significant change at the 5\% level in the rains. It showed just a negative tendency in the number of days without rain (decrease of 0.75 day/year), which possibly was caused by La Niña influence. According to the Mann-Kendall sequential test, the beginning of the decay of rainfall coincided with the year of the increase of the anthropic activities in the region.
\end{abstract}

Keywords: Mann-Kendall, climatological variability, precipitation, semi-arid.

\footnotetext{
R E S U M O

As mudanças atmosféricas ocasionadas pelo processo de desenvolvimento e industrialização afetam diretamente o ciclo hidrológico. A deteç̧ão de tendências em séries temporais de dados hidrológicos é de grande importância científica e prática para a gestão dos recursos hídricos. O objetivo deste estudo é averiguar as tendências e as flutuações ao longo do tempo das variáveis climatológicas (precipitação diária máxima anual; precipitação total anual; dias sem chuva; e precipitação das estações do ano) no município de Toritama-PE. Foi utilizado o teste Mann-Kendall e o estimador Sen's Slope $(\beta)$ para determinar a existência de tendência e sua magnitude. Para identificar flutuações nas tendências ao longo do tempo e para detectar os possíveis pontos de mudança na série de chuvas se utilizou o teste sequencial de Mann-Kendall. O teste não paramétrico de Mann-Kendall demonstrou não haver mudança significativa ao nível de 5\% nas chuvas em estudo, havendo apenas uma tendência negativa no número de dias sem chuva (decrescimento de 0,75 dia/ano) causada, possivelmente, por influência da La Niña. Segundo o teste sequencial Mann-Kendall, o início do decaimento da atividade pluviométrica coincidiu com o ano do aumento das atividades antrópicas na região. Palavras-Chave: Mann-Kendall, variabilidade climatológica, precipitação, semiárido.
} 


\section{Introdução}

Com o desenvolvimento da sociedade e a industrialização, houve um crescimento do uso de combustíveis fósseis. Como decorrência desse uso crescente, somado às queimadas, uso da terra $\mathrm{e}$ emissão de outros gases, houve mudanças na composição da atmosfera, aumentando a quantidade de gases poluentes de longa vida, sendo o principal deles o $\mathrm{CO}_{2}$ (Lima et al., 2012). Esse aumento traz impactos ao ecossistema e ao ciclo hidrológico, no geral, podendo levar a alteração no regime de chuvas. $\mathrm{O}$ aumento na temperatura global resulta na diminuição da capacidade de retenção na atmosfera, o que aumenta os eventos hidrológicos extremos (Phillip, 2011).

Uma forma de averiguar essas mudanças é através da análise de tendências em séries temporais de diversos fatores climáticos $\mathrm{e}$ hidrológicos, incluindo a precipitação. Esse tipo de procedimento consiste em obter dados históricos da variável em investigação e aplicar os métodos desejados. Esses métodos podem ser os mais diversos: modelos de suavização exponencial, modelos auto regressivos, regressão linear e tecnologias de inteligência computacional (redes neurais, lógica nebulosa, etc.) (Ferreira, 2012).

A detecção de tendências em séries temporais de dados hidrológicos é de grande importância científica e prática para a gestão dos recursos hídricos. Em geral, as obras de engenheira hídrica são dimensionadas a partir de chuvas e vazões de projetos (Hietogramas e Hidrogramas), que por sua vez comumente são desenvolvidos por intermédio de uma análise estocástica das séries históricas de precipitação e vazão extremas, para isso a série utilizada deve ser considerada estacionária, ou seja, não deve existir tendências significativas (Back, 2001). A análise espacial de tendências permite observar as mudanças no comportamento e determinar em quais regiões uma determinada variável vem sofrendo mudanças significativas ao longo do tempo (Salviano et al., 2016).

A precipitação está entre as variáveis climáticas de grande relevância e averiguar seu comportamento em escala regional tem expressiva importância. Segundo Bertoni \& Tucci (1993), o conhecimento do regime pluviométrico é de extrema importância para gerenciar as necessidades de abastecimento doméstico, industrial e de irrigações na agricultura.

Vários estudos com este objetivo foram realizados em diversas cidades no Brasil e ao redor do mundo. Partal \& Kahya (2006) determinaram tendências nas séries de média anual e mensal de precipitação total usando o teste não paramétrico de Mann-Kendall na Turquia. Gocic \& Trajkovic (2013) analisaram tendências anuais e sazonais para temperaturas máximas e mínimas, umidades relativas máximas e mínimas, pressão de vapor, velocidade do vento e precipitação, utilizando os testes de Mann-Kendall e Sen's Slope na Sérvia. Blain (2010) e Penereiro \& Ferreira (2012) analisaram as mudanças no comportamento da precipitação e vazão no estado de São Paulo utilizando análise de regressão, Mann-Kendall e Pettit. Berigo \& Thebaldi (2017) analisaram a tendência temporal da precipitação máxima diária anual, mensal e total anual do município de Formiga - MG, por meio de regressão linear simples e teste de Mann-Kendall.

No Nordeste brasileiro, Lima et al. (2011) observaram mudanças significativas em alguns postos pluviométricos do semiárido baiano. Lopes \& Silva (2013), também no Nordeste, determinaram um aumento em todas as séries na região do Sertão dos Inhamuns (Ceará), exceto no período seco, onde houve diminuição nas precipitações, mas sem significância estatística. $\mathrm{Na}$ Bacia do Rio Capibaribe (Pernambuco) foram analisados os índices de detecção de mudanças climáticas por Assis et al. (2012) e os resultados mostraram mudança no padrão das precipitações observadas, com tendência de redução dos índices pluviométricos, juntamente com a diminuição do número de dias com precipitações intensas e aumento de dias secos. No entanto, a distribuição às estatísticas de Mann-Kendall em uma região não é forte o suficiente para concluir à grande escala regional (Nasri \& Modarraes, 2009), fazendo-se necessária uma análise distinta em cada município.

O município de Toritama, foco desse estudo, está localizado na região semiárida do Agreste Pernambucano, na Bacia do Rio Capibaribe. É uma região que apresenta baixo índice de precipitação (com uma média de 530 $\mathrm{mm} / \mathrm{ano}$ ), onde esse regime de chuvas é altamente concentrado entre março e julho. Além disso, estima-se que $90 \%$ das águas precipitadas nessa região não são aproveitadas (Conti \& Schroeder, 2013). Desse modo, é uma região com limitação de recursos hídricos, o que torna fundamental uma análise de tendência para entender mudanças de comportamento no clima e possíveis eventos climatológicos extremos. Toritama também é líder em produção têxtil do Polo de Confecções do Agreste de Pernambuco, que tem despontado como importante polo produtor de vestuário, inicialmente ao longo da década de 90 (Araújo \& Pereira, 2006). Dessa maneira, o estudo das 
possíveis escassez de recursos hídricos nessa região torna-se imprescindível.

Diante do exposto, este estudo objetiva avaliar as mudanças climáticas hidrológicas do município de Toritama através de análises de tendência dos dados pluviométricos pelos testes de Mann-Kendall, sequencial Mann-Kendall e Sen's Slope.

\section{Material e Métodos}

Localização da área de estudo

$\mathrm{O}$ estudo foi realizado no município de Toritama, localizado na latitude $7^{\circ} 59^{\prime} 56^{\prime}$ ' S e longitude $36^{\circ} 3^{\prime} 8^{\prime \prime}$ 'W, a uma altitude de $356 \mathrm{~m}$ (Figura 1). Estando localizado na região semiárida do nordeste brasileiro, no estado de Pernambuco, na bacia do rio Capibaribe, o seu clima é classificado por Köppen-geiger como As, possuindo um clima tropical com estação seca (DB-CITY, 2017).

\section{Dados Pluviométricos}

Os dados pluviométricos utilizados foram obtidos na Agência Pernambucana de Águas e Climas (APAC, 2018), sendo eles referentes ao posto pluviométrico de código $70 \mathrm{com}$ uma série histórica de 1963 a 2017.

Neste estudo foram definidos quatro parâmetros a serem analisados sob o aspecto de mudanças climáticas, sendo esses: precipitação total anual, precipitação diária máxima anual, número de dias sem chuva, e as estações do ano (Verão, Outono, Inverno e Primavera). As estações do ano foram definidas de modo semelhante ao da APAC (2018), onde o acumulado dos três primeiros meses (janeiro a março) é denominado Verão, os três subsequentes meses (abril a junho) é o Outono, o Inverno é considerado no intervalo entre julho e setembro, e os últimos meses (outubro a dezembro) é a Primavera.

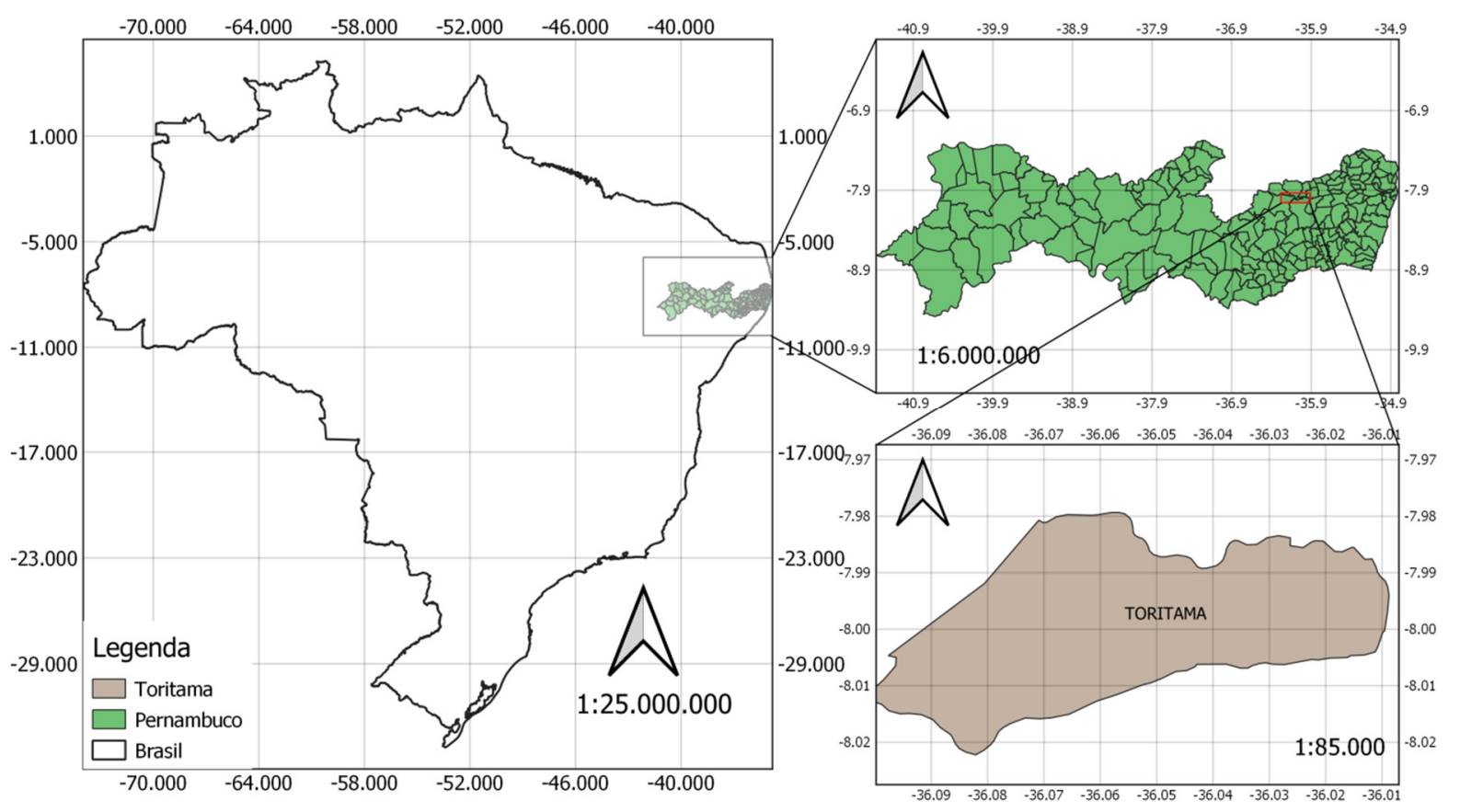

Figura 1 Mapa de Localização do município de Toritama-PE. Fonte: Alcântara et al. (2018).

\section{Metodologia Mann-Kendall}

Para avaliar as mudanças climáticas na região foi utilizado o teste não paramétrico de Mann-Kendall (MK). Este teste, junto à estatística de correlação de Tau de Kendall $(\tau)$, é comumente utilizado para avaliar a tendência de dados ambientais, climatológicos e hidrológicos (Asfaw et al., 2018). Nele, a hipótese nula $\left(\mathrm{H}_{\mathrm{o}}\right)$ é adotada como um conjunto de variáveis aleatórias independentes e distribuídas identicamente. Enquanto a alternativa $\left(\mathrm{H}_{1}\right)$ assume a não identicidade de $X_{k}(k=1,2, \ldots . j-1)$ e $X_{j}(j=1,2, \ldots n)$ para um teste bilateral (Partal \& Kahya, 2006). O teste $\mathrm{Z}_{\mathrm{MK}}$ é calculado baseado em Mann (1945) e Kendall (1948) usando a seguinte equação:

$\mathrm{Z}_{\mathrm{MK}}=\left\{\begin{array}{lc}\frac{\mathrm{S}-1}{\sqrt{\mathrm{Var}}}, & \text { se } \mathrm{S}>0 \\ 0, & \text { se } \mathrm{S}=0 \\ \frac{\mathrm{S}+1}{\sqrt{\mathrm{Var}}}, & \text { se } \mathrm{S}<0\end{array}\right.$

onde:

$\mathrm{S}=\sum_{\mathrm{k}=1}^{\mathrm{n}-1} \sum_{\mathrm{j}=\mathrm{k}+1}^{\mathrm{n}} \operatorname{Sign}\left(\mathrm{X}_{\mathrm{j}}-\mathrm{X}_{k}\right)$ 
onde:

$\operatorname{Sign}\left(X_{j}-X_{k}\right)=\left\{\begin{array}{c}-1,\left(X_{j}-X_{k}\right)<0 \\ 0,\left(X_{j}-X_{k}\right)=0 \\ +1,\left(X_{j}-X_{k}\right)>0\end{array}\right.$

onde: $\mathrm{X}_{\mathrm{j}}$ e $\mathrm{X}_{\mathrm{k}}=$ valores anuais dos dados em j e $\mathrm{k}$, respectivamente; $\mathrm{n}=$ número total $\mathrm{de}$ anos estudados.

Tem sido documentado para observações maiores que 10 anos $(n>10)$ que a estatística 'S' é aproximadamente normal com média e esperança E(S) iguais à zero (Kendall, 1948). Neste caso, a variância é dada por:

$$
\operatorname{Var}(\mathrm{S})=\frac{n(n-1)(2 n+5)-\sum t(t-1)(2 t+5)}{18} \quad \text { Eq. }(4)
$$

onde: $\mathrm{n}=$ número de termos; $\mathrm{t}=$ número de termos iguais.

Segundo Blain (2010), $\mathrm{H}_{\mathrm{o}}$ não é rejeitada, a um nível de significância $(\alpha)$ de $5 \%$, se o valor absoluto de $\mathrm{Z}_{\mathrm{MK}}$ (equação 1) for menor que 1,96, valor determinado a partir da tabela da distribuição normal reduzida. A tendência é classificada como positiva ou negativa de acordo com o sinal do parâmetro $\mathrm{Z}_{\mathrm{MK}}$.

O coeficiente $\tau$ mede a magnitude da relação entre x e y (Bari et al., 2016). A correlação de Tau de Kendall pode ser obtida por:

$$
\tau=\frac{S}{n(n-1) / 2}
$$

\section{Sen's Slope}

O teste Sen's Slope é utilizado para se calcular a magnitude das tendências. Para $\mathrm{o}$ cálculo de Sen's slope, computam-se todas as curvaturas de todos os pares dos valores apresentados em determinada série temporal. É um método insensível a outliers e dados ausentes, sendo mais rigoroso do que a curvatura da regressão linear, provendo uma medida mais real das tendências em séries temporais. A curvatura de Sen's Slope é obtida pelo cálculo dos pares de valores da série com a aplicação da Equação 6:

$$
\beta=\text { Mediana }\left(\frac{X i-X j}{j-i}\right)
$$

onde $\mathrm{X}=$ precipitação correspondente ao ano $\mathrm{j}$ e $\mathrm{i}$, sendo $\mathrm{j}$ sempre menor que $\mathrm{i}$.

\section{Teste sequencial de Mann-Kendall}

Para determinar o ano aproximado que inicia uma mudança de tendência significativa é utilizado o teste sequencial de Mann-Kendall (SQ-
MK), onde é demonstrada a flutuação da tendência ao longo do tempo (Bari et al., 2016). Este teste utiliza uma série progressiva $(\mathrm{u}(\mathrm{t}))$ e uma série oposta a essa $\left(u^{\prime}(t)\right)$. Quando os valores absolutos de $\mathrm{u}(\mathrm{t})$ são maiores que o limite de confiança adotado (95\% neste estudo), a tendência é considerada significativa. $\mathrm{O}$ ponto de intersecção entre as duas séries define o início da tendência, se ele ocorrer dentro dos valores críticos do intervalo de confiança. Nasri \& Modarres (2009) usam os seguintes passos para o cálculo do SQ-MK: 1 . Os valores de $X_{j}$ da série temporal $(\mathrm{j}=1,2, \ldots, \mathrm{n})$ são comparados com $\mathrm{X}_{\mathrm{i}}$ (i $=1,2, \ldots, \mathrm{j}-1)$. Para esta comparação, a quantidade de casos em que $X_{j}>X_{i}$ é determinada e esse valor é denominado nj; 2 . O teste estatístico t é calculado por meio da Equação 7:

$$
t_{j}=\sum_{1}^{j} n_{j}
$$

A média e a variância do teste são:

$$
\begin{gathered}
E(t)=\frac{n(n-1)}{4} \\
\operatorname{Var}(t)=\frac{n(n-1)(2 n+5)}{72}
\end{gathered}
$$

$\mathrm{O}$ valor de $\mathrm{u}(\mathrm{t})$ é calculado por:

$$
u(t)=\frac{t_{n}-E(t)}{\sqrt{\operatorname{Var}(t)}}
$$

De modo semelhante, os valores de $u^{\prime}(t)$ são determinados com os valores da série ordenadas do último para o primeiro ano.

\section{Resultados}

A Figura 2 mostra a evolução dos parâmetros analisados (precipitação diária máxima, número de dias sem chuva e estações do ano) no período de 1963 a 2017 para o município de Toritama-PE. É possível identificar tendências negativas em todas as variáveis analisadas. $\mathrm{Na}$ Figura 2D podem-se observar, em sequência cronológica, valores maiores no outono e menores na primavera; isso resulta em uma concentração maior da precipitação no segundo trimestre anual. 

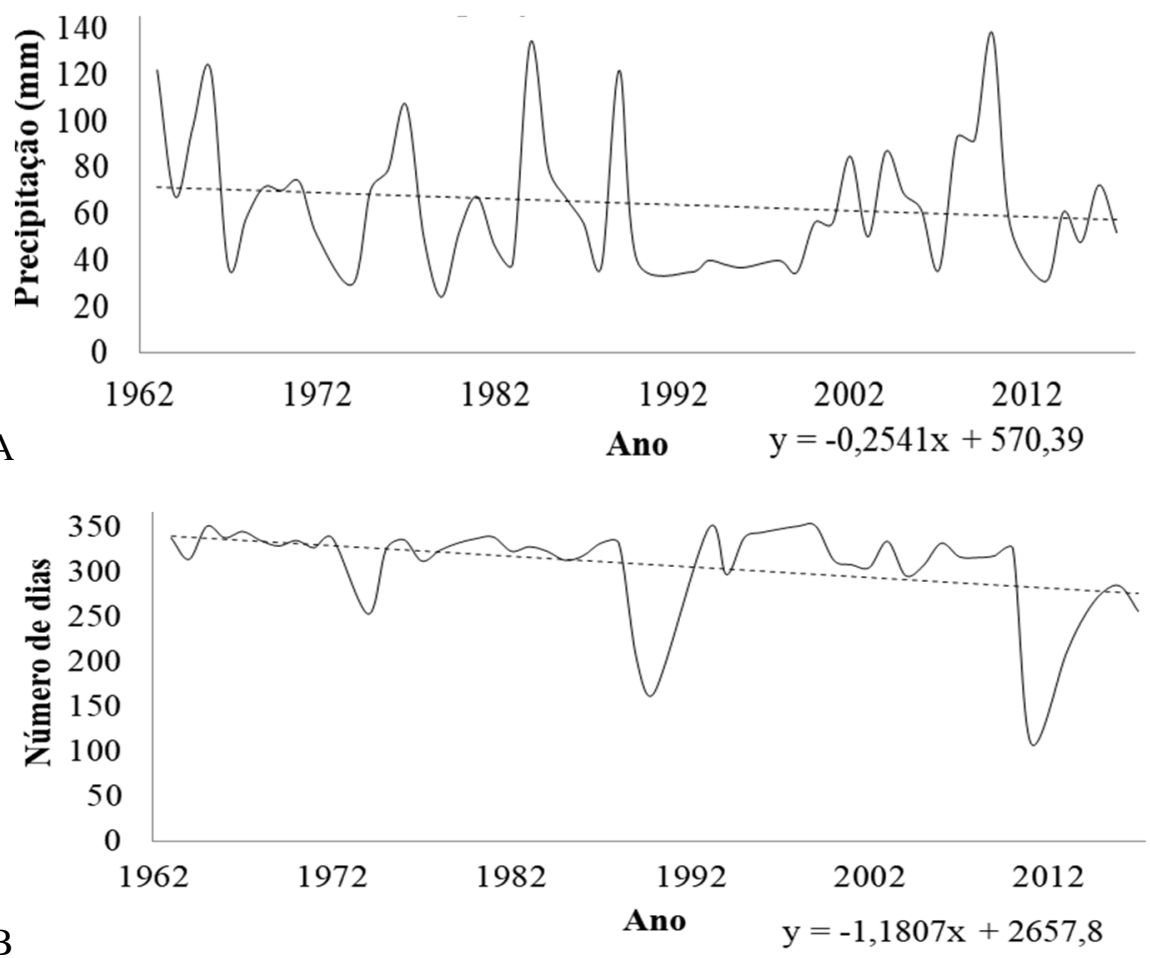

B

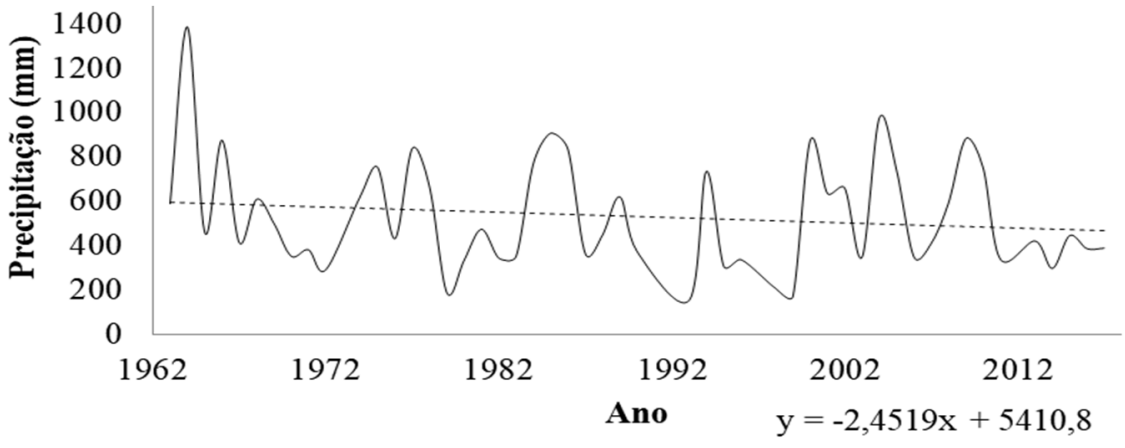

$\mathrm{C}$

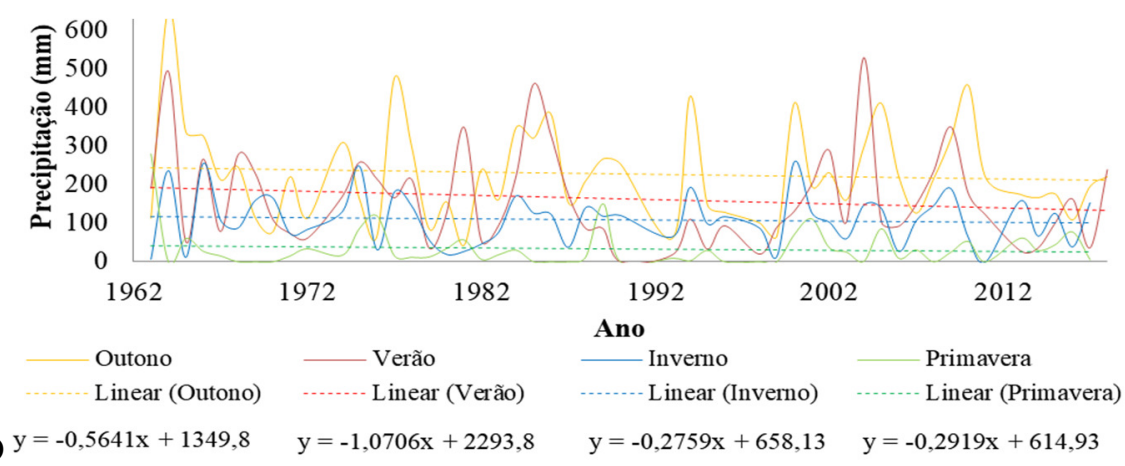

Figura 2. Distribuição dos parâmetros de precipitação em Toritama-PE. A. Precipitação diária máxima; B. Número de dias sem chuva; C. Precipitação total anual; D. Estações do Ano. Fonte: Adaptado da APAC (2018).

A avaliação da distribuição empírica dos dados é realizada através do gráfico BoxPlot (Figura 3). A Figura 3A apresenta um Max Outlier no ano de 2010 e pode ser considerada como simétrica. Na Figura 3B é representado os dias com ausência de chuva, sua distribuição é assimétrica e pode ser identificado um mínimo Outlier pertencente ao ano de 2012. A precipitação anual (Figura 3C) detém uma distribuição assimétrica e um máximo Outlier de $1400 \mathrm{~mm}$. As estações estão demonstradas na Figura 3D, o outono e a primavera apresentam distribuição simétrica e o verão e inverno assimétrica. As estações que apresentam maiores e menores dispersões de valores são outono e primavera, respectivamente. Apenas no inverno não é apresentado Outlier. 
A

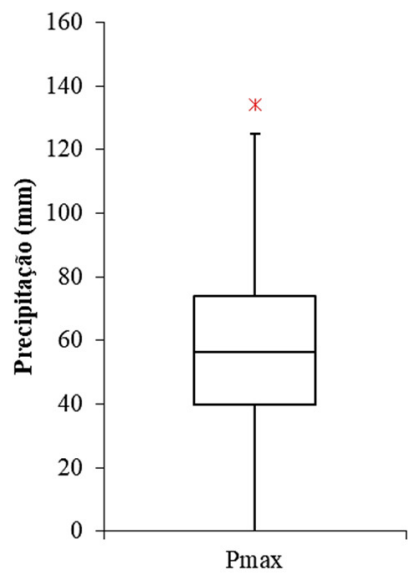

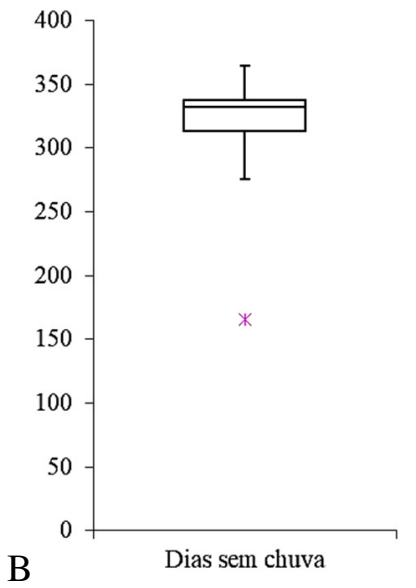

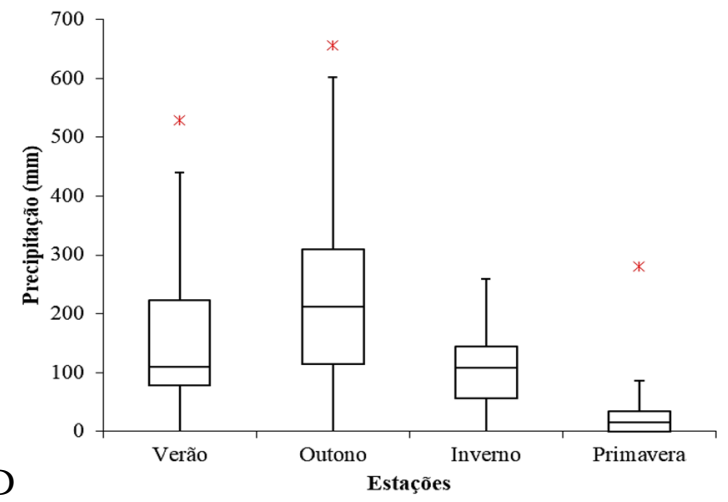

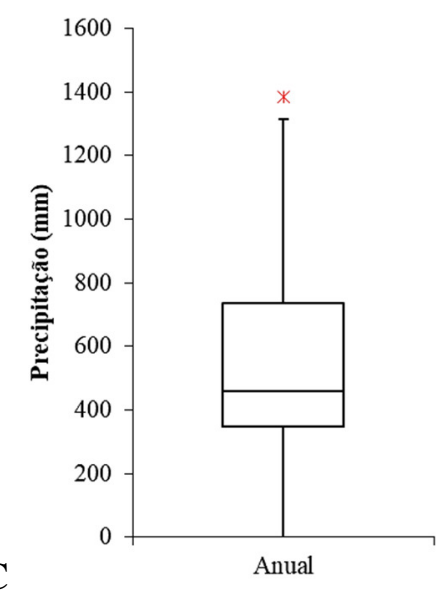

* Min Outlier $*$ Max Outlier

Figura 3. Boxplot dos parâmetros de precipitação em Toritama-PE. A. Precipitação diária máxima; B. número de dias sem chuva; C. Anual; D. Estações do ano. Fonte: Alcântara et al. (2018).

As estatísticas do teste MK são apresentadas na Tabela 1. Existe uma tendência negativa (significativa a 95\%) apenas para o número de dias sem chuva; os demais parâmetros não apresentaram mudanças abruptas. Em sua maioria, os parâmetros mostram ter uma taxa de crescimento negativa em relação à precipitação, com exceção da Primavera que não apresenta mudanças no comportamento de chuvas (0 $\left.\mathrm{mm} \cdot \mathrm{ano}^{-1}\right)$. Mesmo não apresentando tendências no teste de Mann-Kendall, a precipitação anual apresenta o maior decréscimo encontrado pelo Sen's Slope, de aproximadamente 1,65 mm.ano ${ }^{-1}$. Além disso, a relação da tendência com o tempo, medida pelo valor tau de Kendall, foi considerada fraca para todos os parâmetros analisados.

Tabela 1. Resultados dos testes estatísticos de Mann-Kendall e Sen's Slope. *Significativo ao nível de 5\%.

\begin{tabular}{lccccc}
\hline Parâmetro & $\mathbf{S}$ & $\mathbf{V a r}$ & $\mathbf{Z}_{\mathbf{M K}}$ & $\boldsymbol{\tau}$ & $\boldsymbol{\beta}$ \\
\hline Anual & -118 & 15157.33 & $-0,95033$ & $-0,09255$ & $-1,64688^{*}$ \\
Verão & -113 & 16058.33 & $-0,88383$ & $-0,08522$ & $-0,90193^{*}$ \\
Outono & -27 & 16058.33 & $-0,20517$ & $-0,02036$ & $-0,19091^{*}$ \\
Inverno & -29 & 15156.33 & $-0,22744$ & $-0,02275$ & $-0,14333^{*}$ \\
Primavera & 43 & 14887.67 & 0,34422 & 0,033725 & $0,00000^{*}$ \\
Máxima & -125 & 15151.67 & $-1,00738$ & $-0,09804$ & $-0,23636^{*}$ \\
Dias Sem chuva & -414 & 15133.33 & $-3,35724^{*}$ & $-0,32471$ & $-0,75000^{*}$ \\
\hline
\end{tabular}

$\mathrm{O}$ teste sequencial de Mann-Kendall é apresentado na Figura 4. No verão (Figura 4A) é observado um decréscimo significativo no ano de 1998, sendo este iniciado em 1995 (ponto em que os gráficos se cruzam), porém retoma o crescimento no ano 2000. As precipitações do outono e inverno (Figura 4BC) não apresentaram mudanças significativas no comportamento da chuva no período analisado, visto que as curvas não atingiram o limite de significância. As chuvas na primavera e aquelas relativas anuais (Figura 4DE) permaneceram constante até o ano de 1998, voltando ao normal em 2000, pontos onde elas cruzam os eixos limites. Na Figura $4 \mathrm{~F}$ é possível observar a ocorrência de um decréscimo iniciado em 1985, sendo significativo entre 1993 e 2003 . A 
intensificação da escassez hídrica que ocorreu nos anos observados pode ser resultado do El Niño, o qual é caracterizado por provocar secas intensas no Nordeste, que afetam, principalmente, a região semiárida. É notório o paralelismo entre as duas curvas na Figura 4G (dias sem chuva), indicando
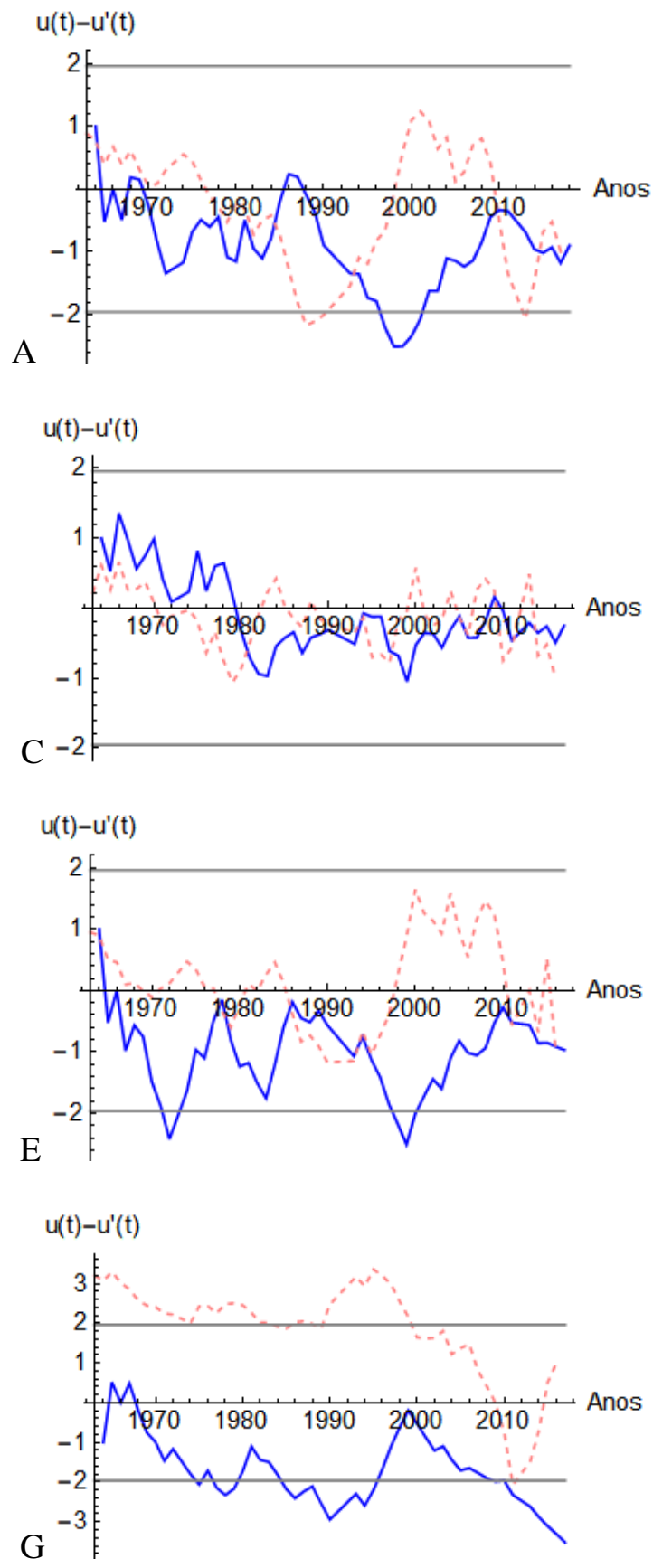

que não ocorreria diferença significativa nesse parâmetro com o decorrer do tempo, porém, conforme as observações vistas na Figura 2B, os eventos extremos em 1972, 1992 e 2012 influenciam em uma mudança de tendência significativa.
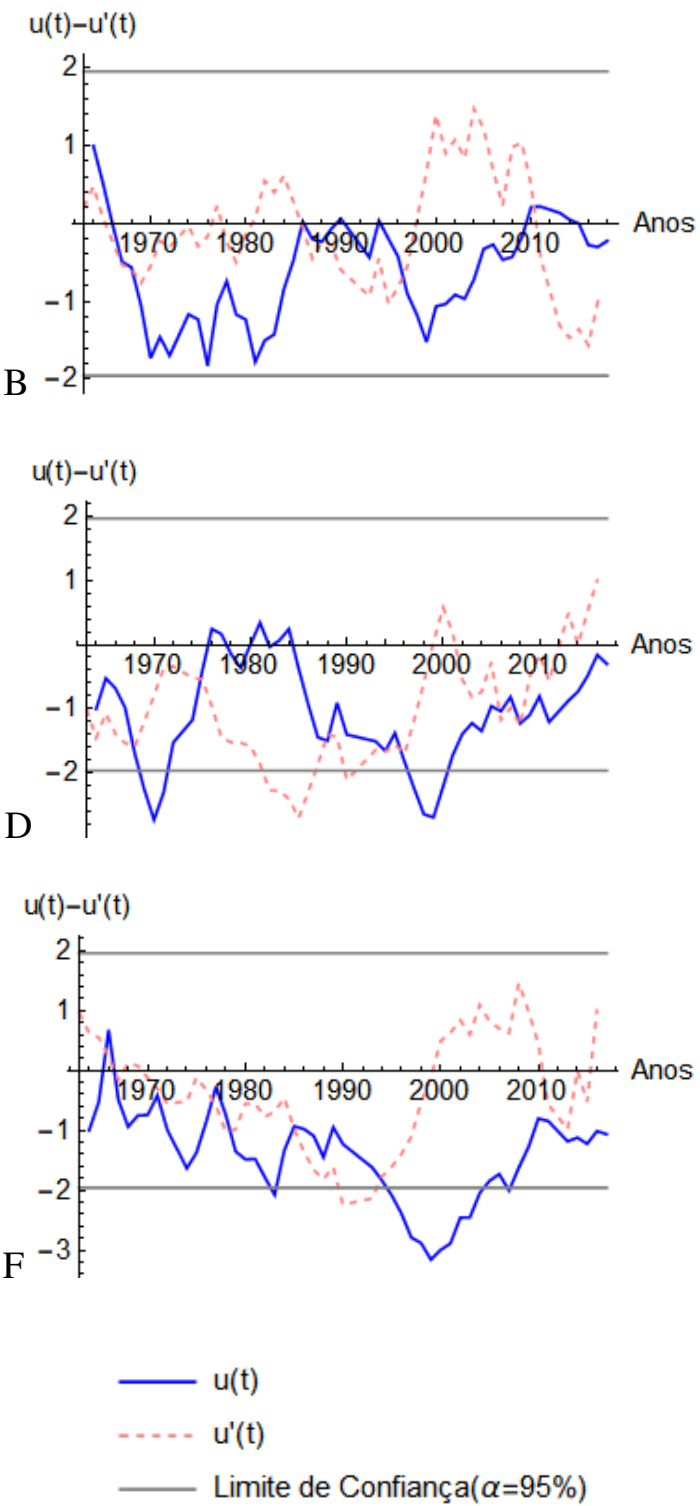

Figura 4. Teste Sequencial de Mann-Kendall (SQ-MK), com limite de confiança a 95\%. A. Verão; B. Outono; C. Inverno; D. Primavera; E. Anual; F. Precipitação diária máxima; G. número de dias sem chuva. Fonte: Alcântara et al. (2018).

\section{Discussão}

Todas as análises por linha de tendência se apresentaram decrescentes, resultados como esse foram obtidos por Jhajharia et al. (2012), no Nordeste da Índia, e por Lopes \& Silva (2013), na região semiárida do Ceará. O número de dias sem chuva apresenta decaimentos no ano de 1972,
1992 e 2012, indicando que em um tempo de retorno aproximado de 20 anos ocorrem maiores durações de chuvas no município de Toritama. Esse fenômeno é possivelmente associado ao evento La Niña que é responsável pelo aumento de precipitação e vazões dos rios dessa região (INPE, 2019). 
A precipitação máxima se apresenta próxima de uma distribuição normal, isso vai contra o que foi exposto por Naghettine \& Pinto (2007), que afirma que a distribuição de variáveis aleatórias com melhor adequação a valores hidrológicas extremos é a de Gumbel.

Com o teste de Mann-Kendall foi possível observar todos os parâmetros de chuva com uma tendência de decrescimento insignificantes, com exceção do número de dias sem chuva, o qual deteve significância ao nível de 5\%. Ao utilizar Mann-Kendall, Lima et al. (2011) também obtiveram tendências negativas não significativas na maioria das localidades pertencentes ao semiárido da Bahia. O mesmo ocorreu com Lopes \& Silva (2013) no semiárido do Ceará. Esse fato indica que o semiárido Nordestino está, possivelmente, passando por uma diminuição no seu regime pluviométrico, mas que, atualmente, ainda pode ser considerada significante. Em relação ao número de dias secos, Nasri \& Modarraes (2009) também observaram uma tendência negativa na província de Isfahan no Irã (onde também é uma região semiárida), porém essa tendência não foi considerada significativa.

Foram observadas mudanças na tendência no Verão, Primavera, Precipitação Máxima Diária Anual e Precipitação Total Anual pelo teste sequencial de Mann-Kendall por volta da década de 90. Isso também foi observado por Nasri \& Morradaes (2009), em que as magnitudes dos períodos de seca de 1980 apresentaram decrescimento, especialmente nas regiões áridas e semiáridas. Penereiro \& Ferreira (2012) também encontraram tendências significativas para a precipitação em Pariquera-Açu (SP) apenas a partir do ano de 1995. Bari et al. (2016) detectaram, no norte de Bangladesh, uma tendência decrescente de chuvas sazonais após o início dos anos 90 para a maioria das estações estudadas. Nunes et al. (2018) observaram uma convergência de mudanças nos índices de eventos extremos no ano de 1988, evidenciando um ano representativo de um período de expressiva expansão urbana na região metropolitana de Belo Horizonte. Sendo assim, essa tendência possivelmente ocorreu por interferência do aumento da atividade industrial na região devido ao seu polo têxtil (Araújo \& Pereira, 2006).

\section{Conclusão}

O presente estudo analisou as tendências do número de dias sem chuva e as precipitações sazonal, anual e diária máxima para a cidade de Toritama, além de suas flutuações ao longo do tempo. $\mathrm{O}$ teste não paramétrico de Mann-Kendall demonstrou não haver nenhuma mudança significativa a 5\% nas chuvas em estudo, havendo apenas uma tendência negativa no número de dias sem chuva, o qual pode ser causado por influência da La Niña. Apesar do teste de Mann-Kendall não apresentar mudanças significativas para a pluviometria anual, o Sen's Slope $(\beta)$ mostra um decaimento bastante significativo, devendo ser considerado em virtude das secas severas na região. Segundo o teste sequencial Mann-Kendall, o ínicio do decaimento da atividade pluviométrica coincidiu com o ano do aumento de atividades antrópicas na região do Agreste. Diante disso, pode-se concluir que ocorre uma redução nos padrões pluviométricos não significativos nas comunidades presentes em Toritama.

\section{Agradecimentos}

O estudo foi realizado com apoio do Conselho Nacional de Desenvolvimento Científico e Tecnológico (CNPq), Processo $\mathrm{N}^{\circ}$ 465764/2014-2, da Coordenação de Aperfeiçoamento de Pessoal de Nível Superior Brasil (CAPES), Processo $\mathrm{N}^{\circ}$ 88887.136369/2017-00 e da Fundação de Amparo à Ciência e Tecnologia de Pernambuco (FACEPE), Processo APQ-0498-3.07/17, através do projeto Observatório Nacional da Dinâmica da Água e de Carbono no Bioma Caatinga (ONDACBC).

\section{Referências}

APAC. Agência Pernambucana de Águas e Clima. Dados do Posto Pluviométrico. Disponível em:

http://www.apac.pe.gov.br/meteorologia/monitora mento-pluvio.php?posto_id=70. Acesso em: 10/09/2018.

ARAÚJO, C. A. L.; PEREIRA, C. F. 2006. A indústria de confecções em Pernambuco: impactos e oportunidades em um cenário pós-ATC (Acordo sobre Têxteis e Confecções). XIII SIMPEPBauru, SP, Brasil, v. 6.

ASFAW, A.; SIMANE, B.; HASSEN, A.; BANTIDER, A. 2018. Variability and tome series trend analysis of rainfall and temperature in northcentral Ethiopia: A case study in Woleka sub-basin. Weather and Climate Extremes, v. 19, n. 1, p. 29-41.

ASSIS, J.; LACERDA, F. F.; SOBRAL, M. C. 2012. Análise de detecção de tendências no padrão pluviométrico na Bacia Hidrográfica do Rio Capibaribe. Revista Brasileira de Geografia Física, v. 5, n. 2, p. 320-331. 
BACK, A. J. 2001. Aplicação de análise estatística para identificação de tendências climáticas. Pesquisa Agropecuária Brasileira, v. 36, n. 5, p. 717-726.

BARI, S. H.; RAHMAN, M. T. U.; HOQUE, M. A.H.; HUSSAIN, M. M. 2016. Analysis of seasonal and annual rainfall trends in the northern region of Bangladesh. Atmospheric Research, v. 16, n. 1, p. 148-158.

BERIGO, J. S.; THEBALDI, M. S. 2017. Análise da tendência temporal da precipitação do município de Formiga-MG. Enciclopédia Biosfera, v. 14, n. 25, p. 36-48.

BERTONI, J. C.; TUCCI, C. E. M. Precipitação. In: TUCCI, C. E. M. (Org.). Hidrologia Ciência e Aplicação. $2^{\mathrm{a}}$. ed. Porto Alegre: Universidade/UFRGS: ABRH, 1993. Cap. 5, pp. 177-231.

BLAIN, G. C. 2010. Detecção de tendências monótonas em séries mensais de Precipitação pluvial do estado de São Paulo. Bragantia, v. 69, n. 4 , p. 1027-1033.

CONTI, I. L.; SCHROEDER, E. O. 2013. Convivência com o Semiárido Brasileiro: Autonomia e Protagonismo Social. Editora IABS. Brasília-DF.

DB-CITY. Toritama. Disponível em: http://pt.dbcity.com/Brasil-Pernambuco-Toritama. Acesso em: 16 nov. 2017.

FERREIRA, V. O. 2012. Análise de tendências em séries pluviométricas: algumas possibilidades metodológicas. Revista GEONORTE, v. 1, n. 5, p. 317-324.

GOCIC, M.; TRAJKOVIC, S. 2013. Analysis of changes in meteorological variables using MannKendall and Sen's slope estimator statistical tests in Serbia. Global and Planetary Change, v. 100, p. 172-182.

INPE. Monitoramento do El Niño durante DJF2019. Disponível em: http://enos.cptec.inpe.br. Acesso em: 03/02/2019.

JHAJHARIA, D.; YADAV, B. K.; MASKE, S.; CHATTOPADHYAY, S; KAR, A. K. 2012. Identification of trends in rainfall, rainy days and $24 \mathrm{~h}$ maximum rainfall over subtropical Assam in Northeast India. Comptes Rendus Geoscience, v. 344, p. 1-13.
KENDALL, M. G. 1948. Rank Correlation Methods. Ed. C. Griffin, $1^{\text {a }}$ Edition.

LIMA, J. G. A.; VIANA, P. C.; WANDERLEY, J. A. C.; MANIÇOSA, R. M; LIMA, R. M. S. 2012. Análise de tendências na temperatura e precipitação em Caicó, Rio Grande do Norte. Agropecuária Científica no Semiárido. Revista UFCG, v. 8, n. 1, p. 34-38.

LIMA, J. R. A.; NEVES, D. J. D.; DE ARAÚJO, L. E.; AZEVEDO, P. V. 2011. Identificação de tendências climáticas no estado da Bahia. Revista de Geografia, v. 28, n. 3, p. 172-187.

LOPES, J. R. F.; SILVA, D. F. 2013. Aplicação do teste de Mann-Kendall para análise de tendência pluviométrica no estado do Ceará. Revista de Geografia, v. 30, n. 3, p. 192-208.

MANN, H. B. Nonparametric tests against trend. 1945. Econometrica: Journal of the Econometric Society, v. 13, p. 245-259.

NAGHETTINI, M.; PINTO, E. J. DE A. 2007. Hidrologia Estatística. CPRM.

NASRI, M.; MODARRES, R. 2009. Dry spell trend analysis of Isfahan Province, Iran. International Journal of Climatology: A Journal of the Royal Meteorological Society, v. 29, p. 14301438.

NUNES, A. A.; PINTO, E. J. A.; BAPTISTA, M. B. 2018. Detection of trends for extreme events of precipitation in the Metropolitan Region of Belo Horizonte through statistical methods. Revista Brasileira de Recursos Hídricos, v. 23, n. 9, p. 113.

PARTAL, T.; KAHYA, E. 2006. Trend analysis in Turkish precipitation data. Hydrological Process, v. 20, p. 2011-2026.

PENEREIRO, J. C.; FERREIRA, D. H. L. 2012. A Modelagem Matemática Aplicada às Questões Ambientais: Uma abordagem didática no estudo da precipitação pluviométrica e da vazão de rios. Millenium, v. 42, n. 1, p. 27-47.

PHILLIP, R., 2011. Kit de Treinamento SWITCH: gestão integrada das águas na cidade do futuro. Módulo 4. Manejo de águas pluviais: explorando opções. 1. ed. São Paulo: ICLEI Brasil, p. 54. 
Journal of Environmental Analysis and Progress V. 04 N. 02 (2019) 130-139

SALVIANO, M. F.; GROPPO, J. D.; temperatura no Brasil. Revista Brasileira de PELlEGRINO, G. Q. 2016. Análise de Meteorologia, v. 31, n. 1, p. 64-73.

tendências em dados de precipitação e 